\title{
Interfacial fracture energy and the toughness of composites
}

\author{
T. U. MARSTON*, A. G. ATKINS, D. K. FELBECK \\ Department of Mechanical Engineering, University of Michigan, Ann Arbor, Michigan, USA
}

The premises upon which prevailing composite toughness theories are based are discussed in the light of observed strength variations in boron-epoxy composites with differing shear strengths of the interfacial bond. None of the extant toughness theories (pull-out, debonding, stress redistribution) successfully predicts the work of fracture of the boronepoxy system. However, incorporation of the work to create new surfaces into the total toughness analysis gives better agreement with experiment, and work of fracture predictions for other sytems, such as carbon-polyester, can also be modified. The approach is more generalized than the Outwater/Murphy debonding explanation for toughness, which in the way usually presented only applies when the filament fracture strain is greater than the matrix fracture strain. The present analysis suggests how to tailor the interfacial shear strength in order to obtain a reasonable toughness yet still maintain strengths of the order of the rule of mixtures.

\section{Introduction}

Fig. 1 shows the experimentally observed relationship between flexural strength and work of fracture for various surface conditions of boron filament in a boron-epoxy composite. The specimens were the Tattersall and Tappin 3-point bending "roof" design [1], with $42 \%$ volume fraction filaments. The interfacial strength was altered by coating the filaments with silicon vacuum grease in a carefully controlled manner. This process, and further mechanical property data, are to be the subject of a forthcoming publication.

An explanation for the behaviour shown in Fig. 1 led us to review the premises upon which prevailing toughness theories are based. Interpretation of the data depends on the complex inter-relations between tensile strength, $\sigma$, interfacial bonding shear strength, $\tau$, and the composite fracture toughness, $R \cdot \uparrow$ As discussed in detail later, it is generally agreed that as $\tau$ decreases, $R$ increases. Also, arguing solely from a Griffith "fracture mechanics" viewpoint, as $R$ increases, $\sigma$ should also increase. However, considerations of "critical transfer length" and filament reinforcement potential, when taken alone, suggest that as $\tau$ decreases ( $R$ increasing), $\sigma$ also must decrease. The missing link in analyses is the precise $(\sigma, R)$ relation for this latter region.

Section 2 of this paper summarizes the extant theories for toughness in composites. None gives good numerical predictions for works of fracture in the boron-epoxy system (see Appendix). This has led to the consideration of additional contributions to toughness in terms of interfacial energies, in Section 3. The total works of fracture suggested by the new treatments are shown in the Appendix to give improved predictions for the boron-epoxy system and also for the carbonpolyester system, where the principal contributor to toughness is known to be pull-out. Section 4 takes the new analysis for total fracture work and gives an explanation for the behaviour shown in Fig. 1. The conditions that give maximum toughness whilst retaining the rule of mixtures are discussed. Section 5 considers the analysis from the design point of view.

*Present address: Combustion Engineering, Chattanooga, Tennessee, USA

tThroughout this paper, the local specific work of fracture referred to one side of the transverse crack $(R)$ is employed and not the fracture work divided by twice the area (commonly called $\gamma$ ), because of the relationships with generalized cracking theories and "fracture mechanics". For example, $R$ may be identified with $G_{\mathrm{c}}$ or $K_{\mathrm{c}}^{2} / E$, etc. $R$ and $\gamma$ are related, of course, by $R=2 \gamma$. 


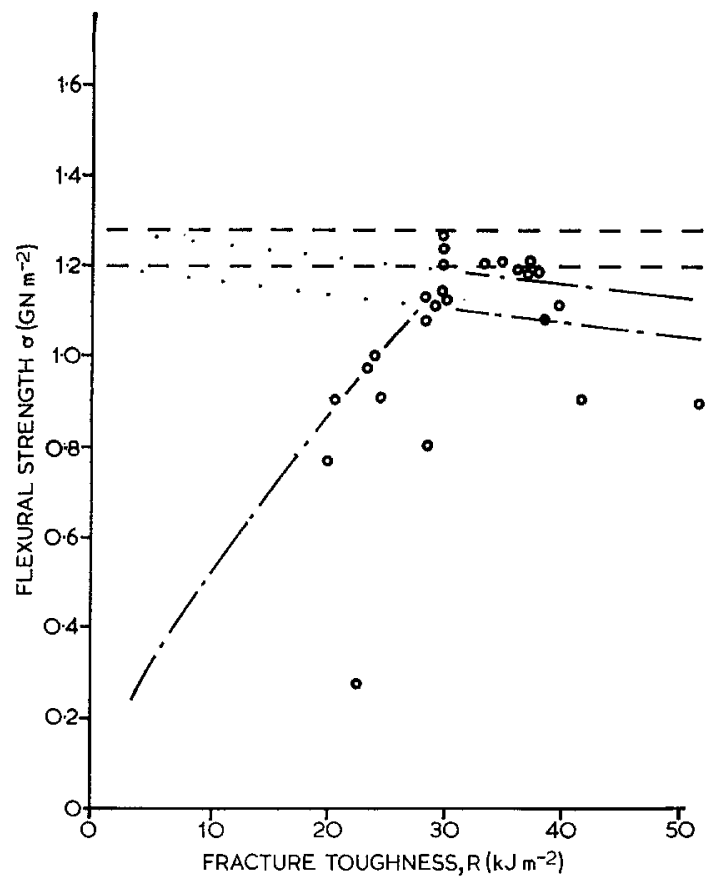

Figure 1 Experimental flexural strengths and toughnesses for Tattersall and Tappin 3-point bending specimens of boron-epoxy.

\section{Present toughness theories}

The mechanisms of "pull-out", "debonding" and "stress redistribution" have so far been put forward to explain the origins of toughness in brittle filament/brittle matrix composites. Analyses bring out the interplay between factors such as interfacial bonding strength and the fracture strength $\left(\sigma_{\mathrm{f}}\right)$, volume fraction $\left(v_{\mathrm{f}}\right)$, diameter $(d)$ and Young's modulus $\left(E_{\mathrm{f}}\right)$ of the filaments, giving the relative importance of these parameters in establishing criteria for tough composites.

\subsection{Pull-out}

The pull-out theory of Cottrell and Kelly (see, for example [2]) attributes the work of fracture to frictional work in pulling broken filaments out of the matrix after fracture. With the assumptions that (i) filaments break randomly, (ii) the original shear strength of the filament/matrix bond is maintained during pull-out and (iii) there is negligible gross plastic flow in the matrix, the specific work of fracture may be expressed as follows:

$$
\begin{aligned}
R_{\text {pull-out }} & =\frac{v_{\mathrm{f}} \sigma_{\mathrm{f}} l_{\mathrm{c}}}{12} \\
& =\frac{v_{\mathrm{f}} \sigma_{\mathrm{f}}^{2} d}{24 \tau}
\end{aligned}
$$

where $l_{\mathrm{c}}=\sigma_{\mathrm{f}} d / 2 \tau$ is the critical transfer length of the filament.

The pull-out theory is successful in predicting toughnesses of metal filament/metal matrix composites, such as tungsten filaments embedded in copper, where the validity of assumption (ii) is demonstrated by copper being smeared on the tungsten filaments after fracture [3].

\subsection{Debonding}

Outwater and Carnes in 1967 developed a different mechanism of toughness specifically for fibreglass systems (see for example Outwater and Murphy [4], by whose names the theory is normally called). Here they attribute the work of fracture to filament debonding, which in fibreglass composites is shown by the relevant areas turning milky. The fracture strain of typical glass filaments is somewhat greater than the fracture strains of typical matrix materials. Glass filaments can thus stretch as a matrix crack passes round them, as shown schematically in Fig. 2. The additional strain energy in the stretched filaments is the source of debond work for glass-resin systems. Debonding progresses until the fracture stress of the stretched filaments is reached.

For reasons that will be apparent later, let us consider the mathematical formulation of the

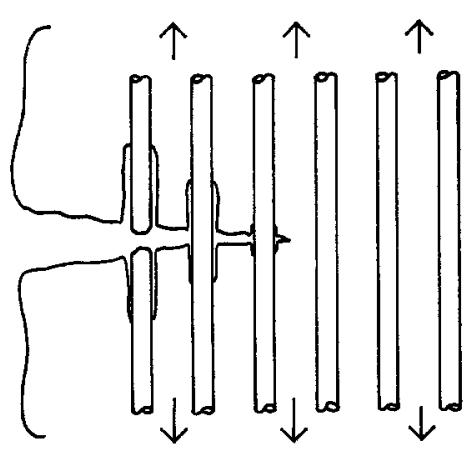

Figure 2 Debonding according to Outwater/Murphy, where the filament fracture strain is greater than the matrix fracture strain, and where the strain energy of the stretched filaments is the source of debond work. No debonding takes place until the crack passes by the filaments. 
Outwater/Murphy theory. The strain energy, $A$, in a length, $L$, of filament under stress, $\sigma$, is

$$
\begin{aligned}
\Lambda= & \frac{1}{2} \frac{\sigma^{2}}{E_{\mathrm{f}}} \text { (stressed volume) } \\
= & \frac{1}{2} \frac{\sigma^{2}}{E_{\mathrm{f}}} \frac{\pi d^{2}}{4} L .
\end{aligned}
$$

From the definition of filament volume fraction,

$$
v_{\mathrm{f}}=\frac{N \pi d^{2}}{4 A}
$$

where $N$ is the number of filaments and $A$ the specimen cross-sectional area.

Thus if debonding occurs on $N$ filaments for an average debond length, $L$, the total strain energy dissipated as work of fracture is

$$
\frac{1}{2} \frac{\sigma^{2}}{E_{\mathrm{f}}} \frac{\pi d^{2}}{4} L \frac{4 A v_{\mathrm{f}}}{\pi d^{2}}
$$

or

$$
\frac{\sigma^{2} L v_{\mathrm{f}} A}{2 E_{\mathrm{f}}}
$$

When $\sigma$ reaches $\sigma_{\mathrm{f}}$ we have

$$
R_{\text {debond }}=\frac{\sigma_{\mathrm{f}}^{2} \eta_{\mathrm{f}} L}{2 E_{\mathrm{f}}} .
$$

Although usually measured experimentally, $L$ must be of the order of $l_{\mathrm{e}}$ if $\sigma_{\mathrm{f}}$ is used in the above derivation, since $L$ on either side of a broken filament has to be at least $l_{\mathrm{c}} / 2$ to attain $\sigma_{\mathrm{f}}$ in the filament (see Fig. 2, and Section 3 following).

Notice that the foregoing is different from the usual debonding mathematics which start by considering stresses within the transfer length. Gurney [5] describes a generalized theory of quasi-static cracking and uses the "embedded rod" model as one illustration. It is shown there that the method of obtaining expressions for toughness by equating the strain energy to the fracture work is strictly erroneous. Rather, the strain energy release rates, as a crack propagates, should be considered. In the case in question, it is fortunate that the answer for toughness is the same by either route. The work of fracture given in this theory is quantified relative to the transverse cross-sectional area of the cracked specimen, and not to the (greater) cylindrical debonded are as. This gives rise to the toughness synergism in composites. If we do divide the work of fracture (Equation 2) by the actual debonded area $(\pi d L)$ we get

*Strictly, only when $L / d \gg 1[5]$.

$$
R_{\mathrm{if}}=\frac{\sigma^{2} d}{8 E_{\mathrm{f}}}
$$

where $R_{\text {if }}$ is the fracture toughness of the filament-matrix interface [5]. $R_{\mathrm{if}}$ is called $W_{\mathrm{d}}$ by Kelly in an "energy equating" derivation [6]. We note that $R_{\text {if }}$ is independent of debond length*; which is of consequence in experiments that measure $R_{\mathrm{if}}$ by debonding. Also, if $R_{\mathrm{if}}>$ $\left(\sigma_{\mathrm{f}}{ }^{2} d / 8 E_{\mathrm{f}}\right)$, the filaments break and no debonding occurs.

Since the energy for debonding, as expressed in the Outwater/Murphy equation (5), must come from additional minute elongations of the fibres, Equation 5 is valid only for those systems where the matrix fracture strain is less than that of the filament. Thus, debonding in the sense envisaged by Cook and Gordon [7] - where a tensile component perpendicular to the fibres in the stress field ahead of a running crack causes interfacial failure before the crack gets to the filament or passes it by (Fig. 3) - cannot be quantified by the Outwater/Murphy approach, since the latter does not allow debonding until after the crack front has passed by the filament. We will return to this point later in the paper.

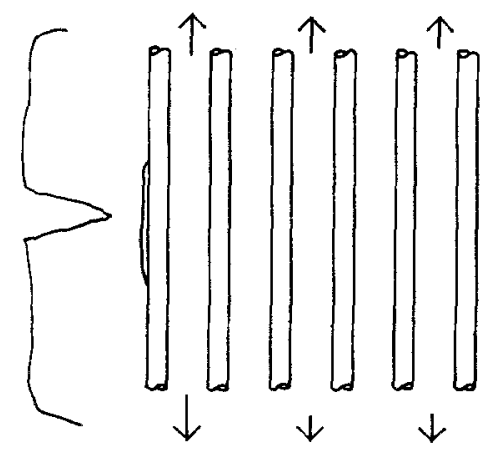

Figure 3 Debonding according to Cook/Gordon, where a tensile component in the stress field ahead of the crack causes interfacial failure before the crack reaches the filament.

\subsection{Stress redistribution}

Piggott [8] and Fitz-Randolph (quoted in [9]) have put forward another explanation for the toughness of composites. The energy-dissipating mechanism is the redistribution of strain energy from the fibre to the matrix after a filament breaks. Before fracture, matrix material adjacent to the impending fracture is virtually unstressed. 
When rupture occurs, the severed ends must pick up load from the matrix, so that the matrix itself picks up load too. The strain energy lost in the fibre is said to be the work of fracture.

Then

$$
\begin{aligned}
R_{\text {redist }} & =\frac{v_{\mathrm{f}} \sigma_{\mathrm{f}}^{2} l_{\mathrm{c}}}{3 E_{\mathrm{f}}} \\
& =\frac{v_{\mathrm{f}} \sigma_{\mathrm{f}}^{3} d}{6 E_{\mathrm{f}} \tau} .
\end{aligned}
$$

Strictly, the net work dissipated should be the loss of filament strain energy less the gain in matrix strain energy, but since typical $\left\{\sigma^{2} / E\right\}_{\mathrm{f}}>$ $\left\{\tau^{2} / G\right\}_{\mathrm{m}}$, where $G$ is the shear modulus, the shear terms may be neglected; we also omit the Poisson's ratio terms of [8].

Notice that the Piggott/Fitz-Randolph $R_{\text {redist }}$ is the direct multiple $\left(2 l_{\mathrm{c}} / 3 L\right)$ of the usual Outwater/Murphy $R_{\text {debond. }}$.

\subsection{Comparison of present theories}

It is clear that these theories will give widely different predictions for $R$. For illustration, if we assume that $\sigma_{\mathrm{f}}=E_{\mathrm{f}} / 100$ roughly, we have $R \mathrm{~s}$ in the ratios 150:3:1 for the Cottrell/Kelly, Outwater/Murphy and Piggott/Fitz-Randolph theories respectively. Nevertheless the analyses can work reasonably well for certain fibre/ matrix combinations. Pull-out has been mentioned in connection with the $\mathrm{W} / \mathrm{Cu}$ system, and it also predicts a large proportion of the work of fracture in carbon fibre/polyester composites. The Outwater/Murphy analysis has found success with glass fibre/resin matrix composites. Fitz-Randolph's theory was proposed for the boron-epoxy system. Careful investigation of the published results [9] shows, however, that in this latter circumstance rather unrealistically long critical lengths were employed to make theory and experiment agree. Critical lengths from 12.5 to $7.75 \mathrm{~mm}$ (which correspond to an interfacial shear strength from 12.5 to $36.2 \mathrm{MN} \mathrm{m}^{-2}$ ) were used, whereas most predictions of the critical length in the boron-epoxy system are of the order of $2.2 \mathrm{~mm}$ and the interfacial shear strength of the order of $65 \mathrm{MN} \mathrm{m}^{-2}[10,11]$.

When applied to composite systems other than those for which they were first used, the three theories are less successful in predicting the toughnesses that are obtained in practice. For example, in a $42 \%$ volume fraction boronepoxy composite, the following fracture toughnesses are predicted: pull-out analysis, 456
$\mathrm{kJ} \mathrm{m}^{-2}$; debonding analysis, $10.8 \mathrm{~kJ} \mathrm{~m}^{-2}$; redistribution analysis, $6.8 \mathrm{~kJ} \mathrm{~m}^{-2}\left(l_{\mathrm{c}}=2.2\right.$ $\mathrm{mm}), 44.4 \mathrm{~kJ} \mathrm{~m}^{-2}\left(l_{\mathrm{c}}=12.5 \mathrm{~mm}\right)$. The experimental toughness for the composite is typically $35 \mathrm{~kJ} \mathrm{~m}^{-2}$ ([11]; see also [9]).

The obvious conclusion from the foregoing, is that the theories work well when the mechanisms upon which they are based are the principal methods of energy dissipation, but that they can be much in error in general when the assumptions in their derivations are violated. For example, the Cottrell/Kelly theory assumes that the original shear strength of the interface is maintained during pull-out which evidently is not always true, so that rather high values of $R$ are predicted. Again, implicit in the Outwater/ Murphy theory is that the fracture strains of the fibres are greater than the fracture strains of the matrix. Moreover, since none of the three theories that have been reported comes close to predicting the boron-epoxy works of fracture, it follows that certain energy dissipation mechanisms (which have been ignored) are operative in that system. A general theory of composite fracture might be expected to incorporate many possible modes of energy dissipation, some of which may be mutually exclusive.

\section{New theory}

\subsection{Background}

Scanning electron microscopy studies which are reported by Marston [11] shed some light on the fracture mechanisms in boron/epoxy composites and the reasons for the inability of the extant theories to predict $R$. Firstly, no interfacial debonding appears to take place prior to filament fracture. Secondly, after filament fracture, the matrix material relaxes away from the filaments thus presumably reducing the normal force at the filament/matrix interface and, therefore, diminishing the frictional force opposing pull-out. These two observations refute the toughness mechanisms of Outwater/Murphy and Cottrell/Kelly. Yet, since the Piggott/FitzRandolph theory does not successfully predict $R$ for this system (using normal $l_{\mathrm{c}}$ ), explanation has to be sought elsewhere.

The new theory suggested in this paper takes into account the contribution of the work done in creating new surfaces during fracture of a composite. Three types of surface are generated, namely: (i) filament cross-sectional surfaces after fracture, (which are generally conical in $\mathrm{B} / \mathrm{W}$ filaments), (ii) matrix cross-sectional 
surfaces after passage of the fracture crack, (iii) cylindrical surfaces created between filament and matrix. The third type of surface is essentially the same as a debonded surface, for which the Outwater/Murphy analysis might be thought to apply. However, their sums are applicable only when the work is supplied from strain energy in the filaments. This is not necessarily the only source of energy and it seems better to develop the argument for total work of fracture in terms of the fracture toughness of the interfacial cylindrical surface. As alluded to in Section 2.2, when a debond is produced by interchange of energy with the filament there must be a relationship between the filament diameter, stress, filament modulus and the interfacial fracture toughness (i.e. the Outwater/Murphy mathematics), but when debonding is produced by other means, such as a Cook/Gordon-type mechanism, there need be no obvious relationship. Yet, since energy is dissipated, it is necessary to be able to express that work in known terms. The proposed method does this; if filament strain energy is the source of debond work, Outwater/ Murphy appears as a special case.

\subsection{New expression}

The theory is developed in detail in the Appendix. The total fracture work is the sum of the works of fracture of the three types of surfaces mentioned above: filament, matrix and filamentmatrix interface. The fracture toughness from this generation of new surfaces is thus the total fracture work divided by the nominal transverse fracture area (i.e. neglecting cylindrical interfacial areas).

We have from Equation A12 in the Appendix,

$$
\begin{gathered}
R_{\text {surfaces }} \approx\left(1-v_{\mathrm{f}}\right) R_{\mathrm{m}}+v_{\mathrm{f}} \frac{l_{\mathrm{c}}}{d} R_{\mathrm{if}} \\
\approx\left[\left(1-v_{\mathrm{f}}\right)+v_{\mathrm{f}} \frac{l_{\mathrm{c}}}{d}\right] R_{\mathrm{m}} \begin{array}{l}
\text { (see assumptions } \\
\text { below) }
\end{array} \\
\approx v_{\mathrm{f}}\left[\frac{l_{\mathrm{c}}}{d}-1\right] R_{\mathrm{m}} \\
\approx v_{\mathrm{f}} \frac{l_{\mathrm{c}}}{d} R_{\mathrm{m}} .
\end{gathered}
$$

Notice that this contribution to work of fracture involves $R_{\text {if }}$ and $R_{\mathrm{m}}$, which are not present in earlier analyses. The physical picture for the "modifying factor" $l_{\mathrm{c}} / d$ (or $\left.\sigma_{\mathrm{f}} / 2 \tau\right)$, which has a pronounced effect on $R$, relates to the lengths of the debonded areas.
Some important points of argument used in obtaining the final expression are as follows.

1. The fibres fracture randomly so that the average length of exposed filament on either side of the filament fracture is $l_{\mathrm{c}} / 4$. This is reflected in experimental pull-out lengths in the boronepoxy system, where the longest exposed filament is $l_{\mathrm{c}} / 2$ and the shortest zero. The assumption of random fracture, which has been observed experimentally [11], is also borne out by Kelly's postulate (in [6]) that if the elastic shear strain energy stored in the matrix at the time of fracture is much less than the filament strain energy, then the fibres break randomly. This is expressed by

$$
\tau<\sigma_{\mathrm{f}} \sqrt{ }\left(G_{\mathrm{m}} / E_{\mathrm{f}}\right)
$$

where $G_{m}$ is the matrix shear modulus. Approximate values for the boron-epoxy system are

$$
\begin{gathered}
\sigma_{\mathrm{f}}=3 \mathrm{GN} \mathrm{m}^{-2} \quad E_{\mathrm{f}}=380 \mathrm{GN} \mathrm{m}^{-2} \\
G_{\mathrm{m}}=4.6 \mathrm{GN} \mathrm{m}^{-2}
\end{gathered}
$$

so if $\tau<0.3 \mathrm{GN} \mathrm{m} \mathrm{m}^{-2}$, random filament fractures will occur. The highest observed shear strength is perhaps $0.1 \mathrm{GN} \mathrm{m}^{-2}$ [12].

2. The fracture toughness of the boron filaments is low $\left(\sim 14 \mathrm{~J} \mathrm{~m}^{-2}\right)$ and the net contribution to the total work of fracture from fibre cross-sectional areas may be neglected.

3. The interfacial fracture toughness, $R_{\text {if }}$, between matrix and filament must be known. In the absence of direct information for $R_{\mathrm{if}}$, the toughness of the matrix $R_{\mathrm{m}}$ can be substituted as in Equation $8 \mathrm{~b}$. In assuming $R_{\mathrm{if}} \approx R_{\mathrm{m}}$ we recognize that this provides only an upper bound for the work to generate debonded surfaces. If $R_{\text {if }}>R_{\mathrm{m}}$, matrix material should adhere to the filaments, and where this is observed some value greater than $R_{\mathrm{m}}$ should be used in Equations 8 . A lower bound is not as easily determined, because the filament is normally far stronger in transverse tension than either the matrix or the interface, so the interface could fracture over a wide range of $R_{\mathrm{i} P}$, even with Poisson contraction in the filament. Note that tensile experiments suggested by Equation 6 are successful only when $R_{\text {if }}<\left(\sigma_{\mathrm{f}}^{2} d / 8 E_{\mathrm{f}}\right)$. If, in such tests, filaments fracture with no debonding, a lower bound on $R_{\text {if }}$ is indicated. Clearly for use in Equations 8, the most desirable procedure is to measure $R_{\mathbf{i f}}$ independently.

\subsection{Total fracture work}

When all the mechanisms of toughness related 
earlier are operating, we might expect that the fracture toughness would be given by adding up the separate contributions, namely:

$$
R_{\text {total }}=R_{\text {pull-out }}+R_{\text {redist }}+R_{\text {surfaces. }}
$$

The $R_{\text {debond }}$ of Outwater/Murphy is implicit in $R_{\text {surfaces }}$ and is thus not written separately. When one contributor is not acting (such as $R_{\text {pull-out perhaps in boron-epoxy, see Section }}$ 3.1 ), it is omitted. Numerical examples are given in the Appendix.

\section{Consequences of new analysis}

It is well known that the elementary rule of mixtures for the longitudinal tensile strength of composites implicitly assumes an interfacial shear strength between the filament and matrix at least equal to the shear strength of the weaker component. Associated with strong interfaces is a comparatively low fracture toughness. Weaker interfaces, although diminishing the longitudinal tensile strength, do accommodate more energy dissipation upon fracture of the filament by mechanisms such as those given earlier in this paper.

Increasing $\tau$ from very low values will increase $\sigma$, with an upper limit on $\sigma$ of the rule-ofmixtures value. This follows from the mode of reinforcement. Filament tensile stresses can build up at the ends of the filaments by transfer of shear stresses from the matrix no more rapidly than $\tan ^{-1}(4 \tau / d)$. If $\tau$ is low, the potential strength of the fibres is never reached. Equivalently the critical transfer length $l_{\mathrm{c}}=\sigma d / 2 \tau$ becomes excessively long, longer than either the lengths of given discontinuous fibres or the size of the composite article itself.

Thus the average working stress in the filaments of finite length $L$, is

$$
\sigma_{\mathrm{av}}=\sigma_{\mathrm{f}}\left(1-\frac{l_{\mathrm{e}}}{2 L}\right)=\sigma_{\mathrm{f}}\left(1-\frac{\sigma_{\mathrm{f}} d}{4 \tau L}\right) .
$$

This is the value that would be used in a rule of mixtures calculation, which gives

$$
\sigma=\left(1-v_{\mathrm{f}}\right) \sigma_{\mathrm{m}}+v_{\mathrm{f}} \sigma_{\mathrm{f}}\left(1-\frac{\sigma_{\mathrm{f}} d}{4 \tau L}\right)
$$

as opposed to

$$
\sigma=\left(1-v_{\mathrm{f}}\right) \sigma_{\mathrm{m}}+v_{\mathrm{f}} \sigma_{\mathrm{f}}
$$

for relatively large $\tau$ where $l_{\mathrm{c}} \ll L$.

The relationship in Equation 12 is shown schematically in Fig. $4 a$ and b, where $\sigma$ falls from

the $\mathrm{RoM}$ value with increasing $1 / \tau$ at the rate $[\mathrm{d} \sigma / \mathrm{d}(1 / \tau)]=-v_{\mathrm{f}}\left(\sigma_{\mathrm{f}}{ }^{2} d / 4 L\right)$, which gives $\sigma$ for "weaker" interfaces.
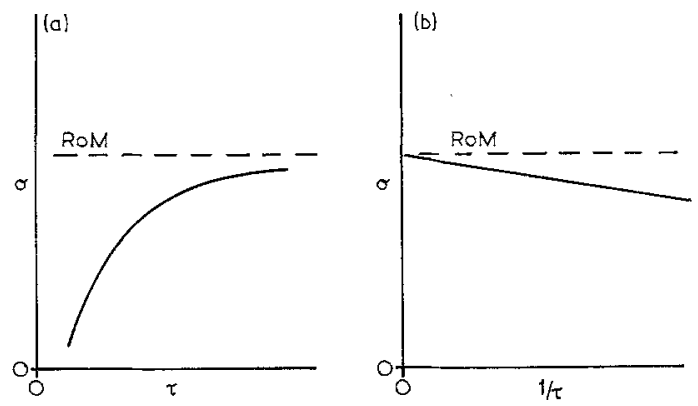

Figure 4 (a) $\sigma$ versus $\tau$ when $\left(\sigma_{\mathrm{f}} d / 4 \tau L\right)$ is not much smaller than unity. (b) $\sigma$ versus $1 / r$ when $\left(\sigma_{p} d / 4 \tau L\right)$ is not much smaller than unity.

The specific work of fracture when we have weak interfaces is given by adding the various contributions due to pull-out, redistribution, creation of new surfaces etc. Thus Equation 10 becomes

$$
\begin{aligned}
& R_{\text {total }}=\frac{v_{\mathrm{f}} \sigma_{\mathrm{f}}^{2} d}{24 \tau}+\frac{v_{\mathrm{f}} \sigma_{\mathrm{f}}^{3} d}{6 E_{\mathrm{f}} \tau} \\
& +\left(1-v_{\mathrm{f}}\right) R_{\mathrm{m}}+v_{\mathrm{f}} \frac{l_{\mathrm{e}}}{d} R_{\mathrm{if}}
\end{aligned}
$$

or, approximately,

$$
\begin{array}{r}
R_{\text {total }} \approx \frac{v_{\mathrm{f}} \sigma_{\mathrm{f}}}{\tau}\left[\frac{\sigma_{\mathrm{f}} d}{6}\left(\frac{1}{4}+\frac{\sigma_{\mathrm{f}}}{E_{\mathrm{f}}}\right)+\frac{R_{\mathrm{m}}}{2}\right] \\
+\left(1-v_{\mathrm{f}}\right) R_{\mathrm{m}} .
\end{array}
$$

Thus, in general terms, $R_{\text {total }}$ varies linearly with $1 / \tau$, with the (small) lower limit on $R_{\text {total }}$ of $\left(1-v_{\mathrm{f}}\right) R_{\mathrm{m}}$ (Fig. 5).* Hence the $1 / \tau$ abscissa of Fig. $4 b$ can be interpreted as an $R_{\text {total }}$ axis. The rate at which $\sigma$ falls with increasing $R_{\text {total }}$ is

$$
\begin{gathered}
\frac{\mathrm{d} \sigma}{\mathrm{d} R_{\text {total }}}=\frac{\mathrm{d} \sigma}{\mathrm{d}(1 / \tau)} \cdot \frac{\mathrm{d}(1 / \tau)}{\mathrm{d} R_{\text {total }}} \\
=-v_{\mathrm{f}} \frac{\sigma_{\mathrm{f}}^{2} d}{4 L} \frac{1}{v_{\mathrm{f}} \sigma_{\mathrm{f}}\left[\frac{\sigma_{\mathrm{f}} d}{6}\left(\frac{1}{4}+\frac{\sigma_{\mathrm{f}}}{E_{\mathrm{f}}}\right)+\frac{R_{\mathrm{m}}}{2}\right]} \\
=\frac{-\sigma_{\mathrm{f}} d}{4 L\left[\frac{\sigma_{\mathrm{f}} d}{6}\left(\frac{1}{4}+\frac{\sigma_{\mathrm{f}}}{E_{\mathrm{f}}}\right)+\frac{R_{\mathrm{m}}}{2}\right]}
\end{gathered}
$$

We seem to have picked up the right hand side * Beaumont and Harris [13] plot essentially $R$ against $\tau$, where $R$ relates principally to pull-out. 


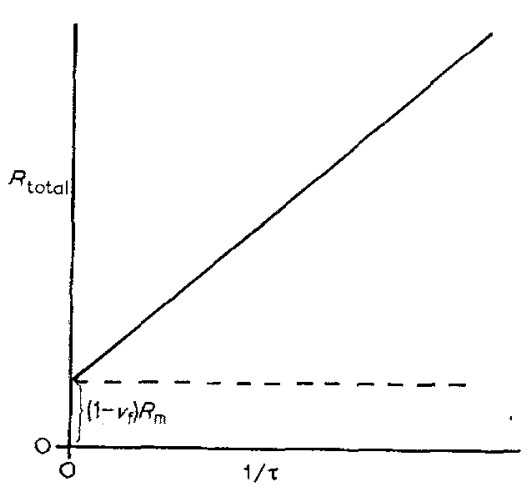

Figure 5 Linear relation between $R_{\text {total }}$ and $1 / \tau$ w ben $\tau$ is "weakened".

of Fig. 1 in this way, where for the boron-epoxy system we would omit the contribution of $R_{\text {pull-out }}$ in scaling between $1 / \tau$ and $R_{\text {total }}$ in Equations 15 and 16.

Regarding the left hand side of Fig. 1, we must remember that the arguments given for $\sigma$ are strictly not valid when bonding is strong enough to satisfy the rule of mixtures assumptions. Then, the composite fracture behaviour is dictated by the propagation of cracks through the brittle matrix, initiated either at flaws in the matrix or by premature low stress failures of weak filaments within the statistical distribution of filament strengths. If we invoke some sort of Griffith argument for the critical crack propagation stress, we have

$$
\sigma^{2} \propto R_{\mathrm{m}} \text {. }
$$

Since we have shown that $R_{\text {total }} \propto 1 / \tau$, then

$$
\sigma^{2} \propto \frac{1}{\tau}
$$

and a plot of $\sigma$ versus $1 / \tau$ or $R$ for the strongly bonded portion of the $1 / \tau$ axis looks as in Fig. 6 . The maximum attainable $\sigma$ is, of course, the RoM value, so the "cut-off" $R$ or $(1 / \tau)$ comes from equating a critical fracture stress formula (from anisotropic fracture mechanics perhaps) with

$$
\left(1-v_{\mathrm{f}}\right) \sigma_{\mathrm{m}}+v_{\mathrm{f}} \sigma_{\mathrm{f}} .
$$

The results for the two regimes in Figs. 4 and 6 are superimposed in Fig. 7, where the $1 / \tau$ abscissa is amended to an equivalent $R$ axis. We see that the experimental $\sigma$ versus $R$ data shown in Fig. 1 follow the general trend in Fig. 7. The right hand slope is given by Equation 16, which

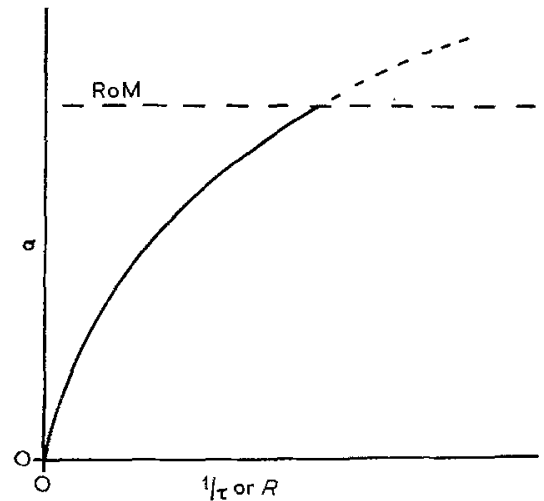

Figure 6 a versus $(1 / \tau)$ or $R$ for strong interfacial bonds.

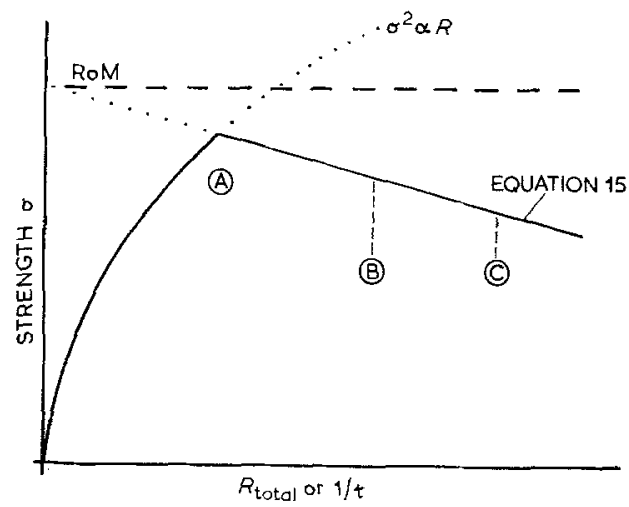

Figure 7 The predictions of Figs. 4 and 6 superimposed, with the abscissa in terms of $(1 / \tau)$ or $R$ via Fig. 5 .

with the boron-epoxy property values in Section 2 of the Appendix becomes - $(44.1 / L) \mathrm{m}^{-1}$. The measured slope from Fig. 1 is, roughly, $-4 \times 10^{3}$ $\mathrm{m}^{-1}$, so that $L \approx 11 \mathrm{~mm}$.

The Tattersall and Tappin bend specimens were $35 \mathrm{~mm}$ long with a $1.6 \mathrm{~mm}$ wide saw cut roof.

\section{Design considerations}

These observations on fracture mode change lead to a unique approach to design for the use of composite materials. From Fig. 7 the designer can select a broad range of strength-toughness combinations. Assuming that he wishes to avoid the relatively steep drop in strength that occurs to the left of point $A$, the designer ought to be able to specify a toughness range, say B-C and thus obtain a known strength range. It is also apparent that a given strength may be achieved from two very different values of toughness, one on either side of point $A$, so a 
strength specification alone is not sufficient. Designers are already acquainted with the strength-toughness trade-offs in selection of martensite tempering temperatures and aluminium alloy ageing temperatures, so the opportunity to make the same sort of choice in composites should present no particular problems.

\section{Conclusions}

The numerical examples in the Appendix show that the proposed expression for total work of fracture, which incorporates debonding in a more generalized way than Outwater/Murphy, and which also involves mechanisms of energy dissipation not hitherto used, is reasonably successful in predicting $R$ for two composites in which the principal contributors to toughness are quite different.

The overall trends given by the expression for fracture toughness agree with observed data between tensile strength and toughness obtained in specimens where the interfacial bonding strength was varied. The conditions for reasonable toughness commensurate with maintaining RoM strengths are contained in the analysis.

\section{Appendix}

\subsection{New analysis}

The new analysis incorporates the energy absorbed in creating all new surfaces during the fracture of a composite material; none of the earlier analyses do this. For the assumptions of the following analysis, and amplification of certain points, Section 3 of the paper should be consulted. In order to predict the total energy absorbed, it is necessary to account for both the area created and the work done to create a unit of that area. The total energy is simply the sum of the products of the areas and their associated energies.

The cross-sectional surface area of newly created filament fractures can be approximated by:

$$
A_{\mathrm{f}}=\frac{N \pi d^{2}}{4}
$$

where $N$ is the total number of filaments fracturing and $d$ is the filament diameter. This assumes a planar type of filament fracture, which is not strictly correct for the double conical fracture present in the boron system. Let us also define $R_{\mathrm{f}}$ as the surface toughness of the filament.
The second type of surface formed is the matrix cross-sectional area which can be approximated by the equation

$$
A_{\mathrm{m}}=A_{\text {nom }}-A_{\mathrm{f}}
$$

where $A_{\text {nom }}$ is the nominal cross-sectional area. This equation neglects small order deviations from a planar fracture surface such as branching, parabola formation, etc. The work of fracture for the matrix material, $R_{\mathrm{m}}$, is determined experimentally with a suitable specimen.

The third type of newly formed surface is that created between filament and matrix as a result of interfacial fractures. They are by far the most important with respect to the dissipation of fracture energy. The surface area created by interfacial fracture can be approximated by:

$$
A_{\text {if }}=N \pi d l
$$

where $l$ is the average exposed length of filament on both halves of the fracture surface. If the filaments fracture randomly, the average length of exposed filament will be one quarter the critical transfer length, since the longest exposed filament will be one half the critical transfer length and the shortest will be zero length.

Thus,

$$
A_{\text {if }}=N \pi d l_{\mathrm{c}} / 4 .
$$

If the energy associated with the interface is $R_{\mathrm{if}}$, the total energy consumed is equal to

$$
\begin{gathered}
U_{\text {total }}=\frac{N \pi d^{2}}{4} R_{\mathrm{f}}+\left(A_{\text {nom }}-\frac{N \pi d^{2}}{4}\right) R_{\mathrm{m}} \\
+\frac{N \pi d l_{\mathrm{c}}}{4} R_{\mathrm{if}} .
\end{gathered}
$$

The fracture toughness is defined as simply the total energy dissipated divided by the area of dissipation or:

$$
\frac{U_{\text {total }}}{A_{\text {nom }}} .
$$

The nominal (or cross-sectional) area can be accounted for in the definition of volume fraction filament:

$$
v_{\mathrm{f}}=\frac{N \pi d^{2}}{4 A_{\text {nom }}}
$$

Rearranging gives:

$$
A_{\text {nom }}=\frac{N \pi d^{2}}{4 v_{\mathrm{f}}} .
$$


Substituting Equations A5 and A8 into Equation A6 gives:

$$
R_{\text {surfaces }}=v_{\mathrm{f}} R_{\mathrm{f}}+\left(1-v_{\mathrm{f}}\right) R_{\mathrm{m}}+\frac{v_{\mathrm{f}} l_{\mathrm{e}} R_{\mathrm{if}}}{d} \text {. }
$$

Using

$$
l_{\mathrm{c}}=\frac{\sigma_{\mathrm{f}} d}{2 \tau}
$$

we get, equivalently,

$$
R_{\text {surfaces }}=v_{\mathrm{f}} R_{\mathrm{f}}+\left(1-v_{\mathrm{f}}\right) R_{\mathrm{m}}+\frac{v_{\mathrm{f}} \sigma_{\mathrm{f}} R_{\mathrm{if}}}{2 \tau} \cdot
$$

If $v_{\mathrm{f}} R_{\mathrm{f}}$ is small,

$$
R_{\text {surfaces }} \approx\left(1-v_{\mathrm{f}}\right) R_{\mathrm{m}}+v_{\mathrm{f}} \frac{l_{\mathrm{c}}}{d} R_{\mathrm{if}}
$$

It is shown in Section 3 of the paper that the significant contribution comes from $v_{\mathrm{f}}\left(l_{\mathrm{c}} / d\right) R_{\mathrm{if}} \approx v_{\mathrm{f}}\left(l_{\mathrm{c}} / d\right) R_{\mathrm{m}}$.

\subsection{Application to boron-epoxy}

Consider the following composite made from "as-received" boron filaments in Shell Epon 828 matrix. $v_{\mathrm{f}}=0.42, \sigma_{\mathrm{f}}=2.96 \mathrm{GN} \mathrm{m}^{-2}, E_{\mathrm{f}}=380$ $\mathrm{GN} \mathrm{m}^{-2}, l_{\mathrm{c}}=2.18 \mathrm{~mm}, d=100 \mu \mathrm{m}, \tau=70$ MN m-2. We do not know the value of $R_{\mathrm{if}}$, but we have measured $R_{\mathrm{m}}$ for Shell Epon 828 epoxy as $2.6 \mathrm{~kJ} \mathrm{~m}^{-2}$ in a 3 -point bend test. Then

$$
\begin{aligned}
R_{\text {total }} & =R_{\text {redist }}+R_{\text {surfaces }} \\
& =6.8+25.1 \\
& =31.9 \mathrm{~kJ} \mathrm{~m}^{-2} .
\end{aligned}
$$

Our experimental values for the as-received filaments were some $35 \mathrm{~kJ} \mathrm{~m}^{-2}$ (cf. Fig. 1). It has been suggested [14] that $2.6 \mathrm{~kJ} \mathrm{~m}^{-2}$ is rather high for $R_{\mathrm{m}}$. Most values reported in the literature are lower [e.g. 13, 15-18], but a comparable value of $\sim 141 \mathrm{~b}$ in. in. ${ }^{-2}$ was measured for an epoxy resin by Fitz-Randolph et al (see [9]). $R_{\mathrm{m}}$ values will depend on the epoxy curing recipe and also on the method of testing (different types of specimen having different strain rate/plane stress/plane strain combinations). Even quite low values of $R_{\mathrm{m}}$ make $R_{\text {surfaces }}$ a significant contributor to $R_{\text {total }}$ in boron-epoxy systems.

\subsection{Application to carbon-polyester}

Consider the following composite [19], $v_{\mathrm{f}}=$ $0.40, \sigma_{\mathrm{f}}=1.6 \mathrm{GN} \mathrm{m}^{-2}, E_{\mathrm{f}}=360 \mathrm{GN} \mathrm{m}^{-2}$, $\tau=20 \mathrm{MN} \mathrm{m}^{-2}, d=8 \mu \mathrm{m}, l_{\mathrm{c}} / d$ (experimental) $=120$, (but note $\sigma_{\mathrm{f}} / 2 \tau=40$ ). In the absence of a value for $R_{\mathrm{m}}$ (replacing $R_{\mathrm{if}}$ ), let us use 600 $\mathrm{J} \mathrm{m}^{-2}$ as a reasonable guess [14].
Then in Equation 10, using $\sigma_{\mathrm{f}} / 2 \tau=40$,

$$
\begin{aligned}
R_{\text {total }} & =R_{\text {pull-out }}+R_{\text {redist }}+R_{\text {surfaces }} \\
& =16.7+0.3+9.6 \\
& =26.6 \mathrm{~kJ} \mathrm{~m}^{-2}
\end{aligned}
$$

or, using $l_{\mathrm{c}} / d=120$,

$$
\begin{aligned}
R_{\text {total }} & =50.4+0.9+28.8 \\
& =80.1 \mathrm{~kJ} \mathrm{~m}^{-2}
\end{aligned}
$$

The experimental values in [19] were about $68 \mathrm{~kJ} \mathrm{~m}^{-2}$. Given the uncertainty about $l_{\mathrm{c}}$ and $R_{\mathrm{if}}$, the agreement seems reasonable. Notice that pull-out, although the greatest contributor to $R_{\text {total }}$, is not sufficient of itself to give the observed fracture toughness.

\section{Acknowledgement}

We are grateful to Dr Bryan Harris for helpful criticism of this paper.

\section{References}

1. H. G. TA Ttersall and G. TAPPIN, J. Mater. Sci. 1 (1966) 296.

2. A. KELLY, in "Strong Solids" (Oxford University Press, 1966).

3. G. COOPER and A. KELLY, ASTM STP-452, 1969.

4. J. O. OU TWATER and M. C. MURPHY, 24th Annual Technical Conference, Composites Division, Society of Plastics Industry, Inc. Paper IIc (1969).

5. C. GURney and J. hunt, Proc. Roy. Soc. (Lond.) A299 (1967) 508.

6. A. KELLY, ibid A319 (1970) 95.

7. J. COOK and J. E. GORDON, ibid A282 (1964) 508.

8. M. PIGGotT, J. Mater. Sei. 5 (1970) 669.

9. P. W. R. BEAUMONT, J. FITZ-RANDOLPH, D. C. phillips and A. S. tetelman, J. Comp. Mater. 5 (1971) 542. See also J. Mater. Sci. 7 (1972) 289.

10. R. A. HeImbuch, Ph.D. Dissertation (1970), University of Michigan, Ann Arbor, Michigan.

11. T. U. MARSTON, Ph.D. Dissertation (1973), University of Michigan, Ann Arbor, Michigan.

12. AVCO Materials Matter 1 (1972) 1.

13. P. W. R. BEA UMONT and B. HARRIS, J. Mater. Sci. 7 (1972) 1265.

14. B. HARRIs, private communication.

15. B. Griffiths and e. holloway, J. Mater. Sci. 5 (1970) 302.

16. F. J. MCGARRY, In "Fundamental Aspects of Fiber Reinforced Plastic Composites", edited by R. T. and H. S. Schwartz (Interscience, New York, 1968).

17. F. J, McG ARRY, Proc. Roy. Soc. (Lond.) A319 (1970) 63.

18. H. T. CORTEN, In "Fundamental Aspects of Fiber Reinforced Plastic Composites", edited by R. T. and H. S. Schwartz (Interscience, New York, 1968).

19. B. HARRIS, P. W.R. BEAUMONT and E. MONCUNILL De Ferran, $J$, Mater. Sci. 6 (1971) 238.

Received 10 July and accepted 24 October 1973. 